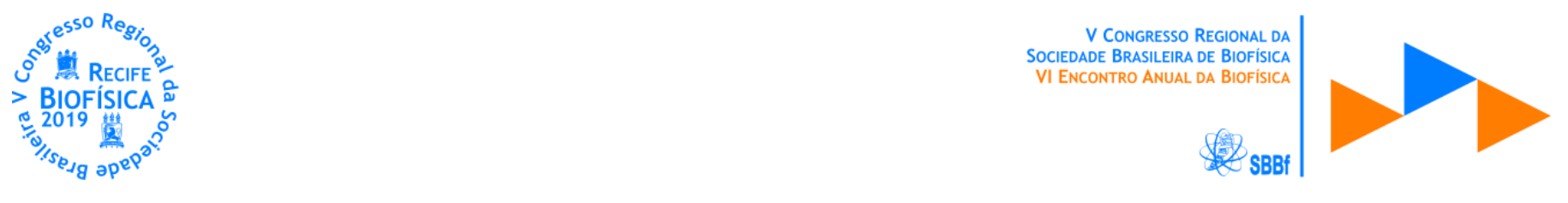

\title{
LIXEIRA INFORMATIVA: A RELAÇÃO DO DADO COM A PRÁXIS AMBIENTAL
}

\author{
Josefa Guedes de Lima Albino ${ }^{1}$, Garuda das Bragas ${ }^{1}$, Valéria Sandra de Oliveira Costa ${ }^{1,2}$, \\ Josicleda Dominciano Galvincio ${ }^{1,2}$, Otacilio Antunes Santana ${ }^{1 *}$ \\ ${ }^{1}$ PROFCIAMB, UFPE; 2PRODEMA, UFPE \\ *otacilio.santana@ufpe.br
}

\begin{abstract}
INTRODUÇÃO
A produção de resíduos urbanos vem a cada ano batendo recordes sem uma expectativa para a redução de seu acúmulo. No mundo, a produção de lixo urbano (doméstico + comércio + repartições públicas, que vão para lixeira) por indivíduo por dia chegou em 2010, a 0,891 kg ind ${ }^{-1} \mathrm{dia}^{-1}$, e em 2018, a 1,127 kg ind ${ }^{-1} \mathrm{dia}^{-1}$ (THE WORD BANK, 2019). No Brasil, esses comportamento é semelhante: $0,714 \mathrm{~kg}^{\text {ind }}{ }^{-1} \mathrm{dia}^{-1}$, em 2010, e 1,218 kg ind ${ }^{-1} \mathrm{dia}^{-1}$, em 2018 (IBGE, 2019). Pode-se com isso estimar que na Região Metropolitana do Recife, com 4.054.866 habitantes, a produção de lixo urbana estaria em torno de 5,5 milhões de tonelada de lixo urbano por dia, o que se faz concordar que essa geração é a geração da produção de lixo "Waste Generation (Era)" (HOORNWEG; BHADATATA; KENNEDY, 2013).

Também para o território brasileiro, estima-se que apenas 13\% dos lixo urbano seja reciclado ou destinado a outro método de redução de seu volume, que não seja seu depósito em aterros e lixões, como por exemplo, compostagem e incineração (IPEA, 2019). No mundo, essa taxa chega a $56 \%$ em país como a Alemanha (SCHUCH et al., 2017), e a 1\%, em país como o Niger (KABERA; WILSON;
\end{abstract} NISHIMWE, 2019).

Pela falta de espaço para estabelecimento de aterros sanitários e de matéria prima para produção de por exemplo embalagens para a indústria alimentícia, ou por seu preço de aquisição (CIACCl et al., 2019), vários países e instituições criaram o movimento "Zero Waste", ou seja, um projeto de ativismo (GROGAN, 1997), ou em alguns casos, um projeto institucional (COSTELLO; MCGARVEY; BIRISCI, 2017), que busca a redução ou erradicação da produção e consumo de resíduos que irão aos aterros e lixões. Seria o estímulo ao consumo de materiais totalmente recicláveis, biodegradáveis e orgânicos.

O projeto "Zero Waste" já foi aplicado em diversos setores estratégicos, com redução entre 50 a $100 \%$ de resíduos destinados aos aterros, como: (a) fashionista (PASRICHA; GREENINGER, 2018); (b) industrial (STEINMETZ et al. 2019); (c) tecnológico (NETO; SOARES, 2018); (d) urbano (FORTUNA; CASTALDI, 2018); (e) hospitalar (SUDARIO, 2018); (f) músico e entretenimento (WHYTE; DIDKOVSKY; HUTZLER, 2018); (g) rural (MANOMAIVIBOOL; SRIVICHAI; UNROJ, 2018); e (h) acadêmico (COSTELLO; MCGARVEY; BIRISCI, 2017).

Em repartições públicas, e principalmente em ambientes acadêmicos, o projeto "Zero Waste" perpassou a função de apenas separação e destinação final do lixo para serem informativos e formativos. Por exemplo, na Universidade de Missouri, nos Estados Unidos, a lixeira informava além do que era para ser depositado nela, para onde iria a destinação final do lixo (COSTELLO; MCGARVEY; BIRISCI, 2017). No Projeto na Universidade de Massey, na Nova Zelândia, a lixeira informava o destino e quanto de dinheiro foi arrecadado com as coletas de reciclados (MANSON et al., 2003).

Além desse caráter informativo, dois projetos que buscam a formação através do engajamento por metas lúdicas são o Projeto "Zero Waste Schools" (NEW YORK CITY, 2019), em Nova lorque, e o "Zero Waste Ragin' Cajun Gameday" (UNIVERSITY OF LOUISIANA AT LAFAYETTE, 2019), em Louisiana, nos Estados Unidos, em que a escola e a universidade estabelecem metas de erradicação do lixo destinado aos aterros, através de competições com a participação da comunidade acadêmica e da comunidade adjacente, com um impacto midiático, e com a presença e atuação de personalidades egressas dessas instituições.

Uma questão de consciência ambiental sobre o lixo é que em geral a maioria da população tem um sentimento que a partir do momento do descarte do resíduos na lixeira e de sua coleta pelo caminhão, esse lixo 'desaparece' (CONNELL, 2010). Apesar de instintivo, não se toma consciência da quantidade de lixo que se é produzido pela sua não visualização. Então, 'produzir mais resíduo para o conforto não é problema, pois ele desaparecerá em breve' (ZANTINGH, 2018).

Com esse intuito, a hipótese desse trabalho foi que a partir da informação da quantidade de lixo que se é produzido por cada indivíduo (tomada de consciência), em um determinado ambiente coletivo, esse próprio indivíduo tentará reduzir a sua produção (tomada de práxis). A coletividade é importante no caso da consciência ambiental, pois os 'mais consciente', por sua atitude, transpõe a práxis ambiental ao outro, ou por meio da educação ou por um 'constrangimento' psicológico sociointeracionista (VYGOTSKY, 1984). E também, com essa coletividade, a ludicidade potencializa processos formativos (SANTANA; PETROVA, 2016).

Os objetivos desse trabalho foram: (a) verificar a demanda de uma lixeira informativa para redução do lixo destinado a aterros e lixões; (b) construir um lixeira biodegradável e informativa; (c) lecionar um curso sobre a importância da informação e a destinação do lixo; (d) implantar as lixeiras em um espaço escolar; (e) diagnosticar a quantidade de lixo produzido e estabelecer metas de redução; (f) sistematizar uma dinâmica lúdica para potencializar as metas; e (g) avaliar, analisar e validar a lixeira e todo processo.

\section{MATERIAL E MÉTODOS}

$O$ procedimento metodológico foi realizado em oito etapas: (a) a quantificação do lixo produzido no espaço escolar; (b) a conscientização dos alunos através de um curso; (c) a construção da lixeira informativa; (d) a implementação das lixeiras; (e) o estabelecimento de metas; (f) a dinâmica lúdica; (g) a validação da lixeira; e (h) a avaliação do processo (Figura 1). 


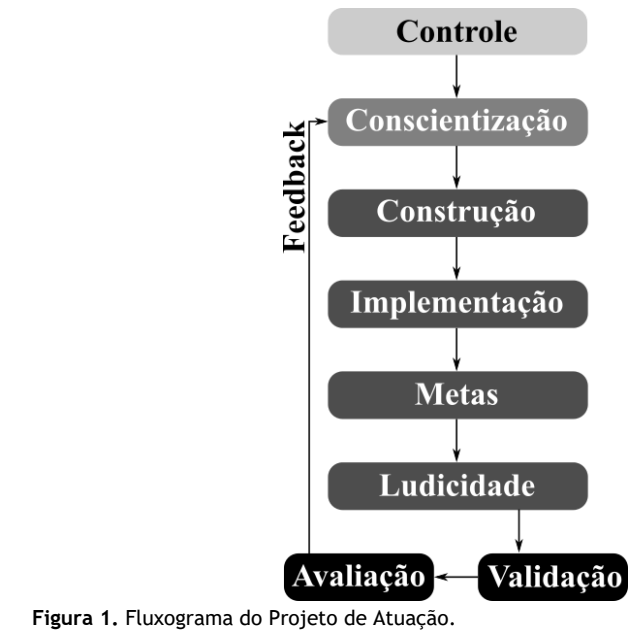

Figura 1. Fluxograma do Projeto de Atuação.

0 estudo foi realizado em uma Escola Estadual de Pernambuco (Código INEP - 26127636), dos anos finais do ensino fundamental ( $6^{\circ}$ ao $9^{\circ}$ ano - 11 a 14 anos) com 447 alunos matriculados e 21 educadores. Aqui por indicação do Comitê de Ética, foi removido o nome da escola, e ao qual, esse projeto de atuação seguiu todas as recomendações do referido Comitê. Esse grupo amostral foi escolhido por compreender um estádio de desenvolvimento cognitivo (Estádio Operatório-Formal) em que a inteligência tornase hipotético-dedutiva e lógica, conseguindo facilmente prever consequências, ou visualizar objetos, sem a necessidade de executar as ações ou que os objetos estejam presentes. Capaz de chegar a unanimidade, através se pontos divergentes, podendo também trabalhar com metáforas. Conseguindo estabelecer relações de cooperação e reciprocidade (PIAGET, 1964).

A demanda de um projeto sobre coleta do lixo foi levantada através de entrevistas não estruturadas com os 21 educadores da escola selecionada (SANTANA, 2014).

A quantificação do lixo produzido $\left(\mathrm{kg}\right.$ aluno $\left.{ }^{-1} \mathrm{dia}^{-1}\right)$ foi realizado através da massa produzida $(\mathrm{kg})$, mensurada diariamente, ao final do expediente, ou quando a lixeira estava completamente cheia, com uma Balança Plataforma 100Kg (Welmy W100/2 Coluna Serial INMETRO, São Paulo, Brasil).

A conscientização do uso da lixeira e da análise semanal das informações inseridas na lixeira foi realizada através de um curso que foi uma ciranda explicativa por educadores de todas as áreas do conhecimento da escola (SANTANA et al., 2015).

A construção da lixeira informativa se deu através do modelo aplicado na Universidade de Louisiana (UNIVERSITY OF LOUISIANA AT LAFAYETTE, 2019), com dois diferenciais (Figura 2): (a) 0 material de sua produção é biodegradável: papelão e fibra de coco e (b) na lixeira possui um painel informativo contendo a quantidade de lixo coletado per capita (estimado pelos presentes nas listas de frequência escolar) e as metas de redução para semana. A impressão das informações e sua confecção se deu em gráficas locais. A capacidade de cada lixeira foi de $100 \mathrm{~L}$, e em cada posição da lixeira na escola, continha três delas identificadas com o material a ser depositado: orgânico, reciclável ou destinado aos aterros e lixões.

As lixeiras foram implementadas em 12 pontos da escola, em seis salas de aulas e seis espaços comuns, divididas em três por blocos (Figura 3), em que cada bloco, os dados foram separados para posterior dinâmica lúdica. A cada semana foi dada a meta de redução de $50 \%$ do lixo que se destina a aterros e $5 \%$ dos outros (recicláveis e orgânicos). Essa meta foi estipulada até a décima semana, para avaliação e retroalimentação do projeto. Nas dez semanas anteriores a implementação da lixeira informativa, foi mensurada a massa do lixo produzido pela escola, e separado manualmente entre: recicláveis, orgânicos e para aterros, a qual foi denominado de Grupo Controle.
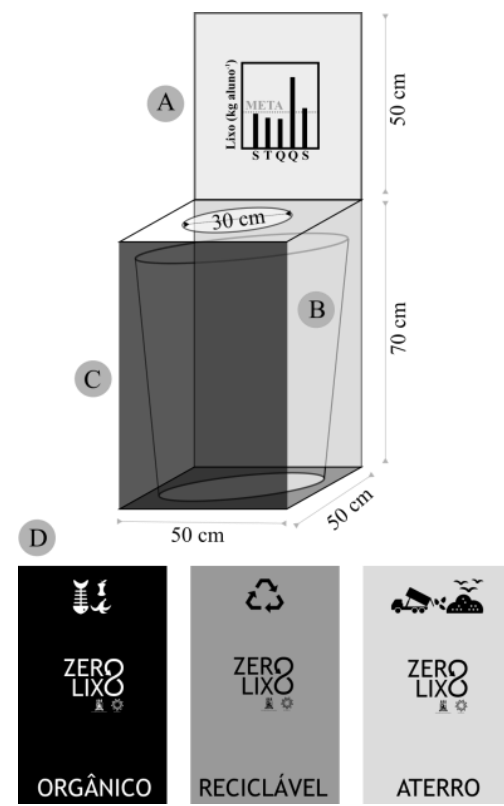

Figura 2. Lixeira informativa: (A) Painel informativo, (B) Recipiente Biodegradável, (C) Presença do Logo e informações do Projeto, (D) Identidade da Lixeira.

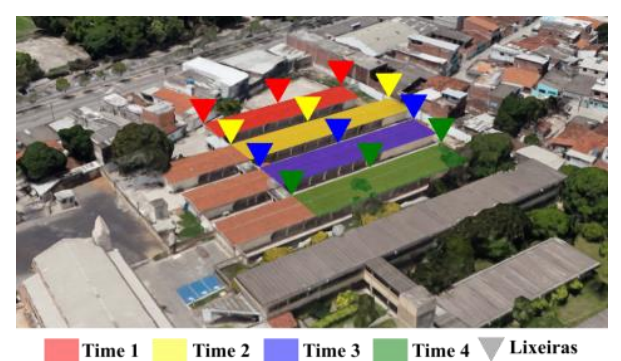

Figura 3. Visão aérea da Escola, a separação dos times por blocos e posição das lixeiras.

Com a lixeira implantada e em uso, dois grupos amostrais foram formados: (a) Grupo das Metas, formado pelos Times 1 e 2 (ver Figura 3), que apenas seguiam as metas estabelecidas semanalmente, e o (b) Grupo Lúdico, formado pelos Times 3 e 4, times que além de seguirem as metas estabelecidas semanalmente, competiam entre si e quem ganhasse, receberia um troféu simbólico 'Certificado de Amigo do Planeta'.

Após, a implementação e aplicação da lixeira, todo o processo foi avaliado pelos 21 educadores, de maneira objetiva, através dos critérios estabelecidos pela Coordenação de Aperfeiçoamento de Ensino Superior para avaliação de produtos técnicos e tecnológicos (CAPES, 2019) em uma escala Likert (1932). Os critérios foram: a) Aderência: se as informações são metas das Ciências Ambientais; b) Impacto: se o produto educacional causou alguma implicação social (impacto ambiental, impacto sanitário, impacto cultural, impacto econômico, etc...); c) Aplicabilidade: se o produto educacional é de fácil manuseio e compreensão, se suas regras e propostas são autoexplicativas, e de utilidade para o público-alvo estabelecido; d) Inovação: se o produto educacional rompe metodologicamente com os recursos didáticos recorrentes; e, e) Complexidade: se o produto educacional é direcionado a uma diversidade de atores, relações e conhecimentos necessários à elaboração e ao desenvolvimento educacional.

A avaliação aberta foi realizada para os educadores exporem quais foram os pontos fortes, fracos, oportunos e ameaçadores (Análise de SWORT, SANTANA et al., 2018). 
As diferenças estatísticas entre os grupos amostrais foram realizadas pelo Teste não paramétrico qui-quadrado $(95 \%$ de confiabilidade) (ZAR, 1999).

\section{RESULTADOS E DISCUSSÃO}

Os dois grupos amostrais Metas e Lúdico obtiveram uma redução significativa $(\mathrm{p}<0,001)$ na produção total de lixo em relação ao Grupo Controle (Figura 4). Os dados do grupo controle ratificam a demanda dos educadores por um projeto de atuação na escola. Com as dinâmicas e implementação da lixeira, o lixo que foi destinado aos aterros reduziram significativamente $(p<0,050)$. Do Grupo Controle para o Grupo Metas, houve um aumento do consumo dos lixos recicláveis e orgânicos $(p<0,001)$. O Grupo Lúdico foi o que teve menor produção de lixo total, em especial para os destinados a aterros e recicláveis, ficando apenas em igualdade estatística com o consumo de orgânicos do Grupo Metas $(p>0,050)$. Os dados amostrados ficaram no alcance $(0,052-0,397$ $\mathrm{kg}$ aluno $\left.^{-1} \mathrm{dia}^{-1}\right)$ do dados apresentados pela literatura $(0,009$ $0,614 \mathrm{~kg}$ aluno $^{-1} \mathrm{dia}^{-1}$ ) para ambientes escolares (KOWALEWSKA; KOLLAJTIS-DOLOWY, 2018; SCHUPP et al., 2018; SHANKS; BANNA; SERRANO, 2017). Em algumas escolas, esse dados se apresenta baixo $\left(\approx 0,150 \mathrm{~kg}\right.$ aluno $\left.{ }^{-1} \mathrm{dia}^{-1}\right)$, pelo sistema de refeição coletiva por 'bandejão' (LIU et al., 2016).

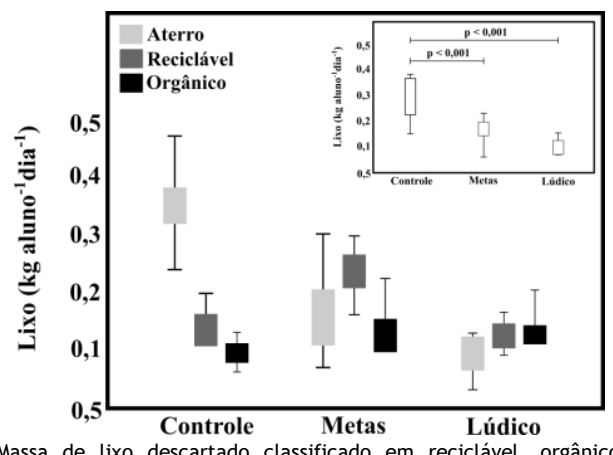

Figura 4. Massa de lixo descartado classificado em reciclável, orgânico e que foi destinado aos aterros, na Escola, nos três grupos amostrais: Controle, Grupo das Metas, e Grupo Lúdico.

Assim, ficou evidente que a lixeira foi efetiva ao expor os dos dados semanais da produção do lixo e o estabelecimento das metas para sua redução. Toda a semana, os Times de 1 a 4 conseguiram cumprir a meta para o lixo que foram para os aterros e lixões. As metas para os lixos reciclados e orgânicos não foram cumpridas, mas entendidas que o seu aumento, ou não redução, foram devidos a direta substituição ao consumo de lixo que resultaria na sua destinação aos depósitos mencionados (Figura 5). Esse comportamento foram observados em outros projetos "Zero Waste", primeiro um aumento da massa de reciclados e orgânicos para depois sua redução (BOEHME; LOGOMARSINO, 2017; ADAMS et al., 2016; MANSON et al., 2003).

Ao longo da observação pelo tempo (Figura 5), as diferenças entre os três grupos corroboram ainda mais com a efetividade da lixeira informativa. O destaque na análise pelo tempo foi o ponto de inflexão no gráfico em que a massa de lixo destinada aos aterros não correspondiam aos maiores valores (Figura 5B e $5 C$ ). Esse momento foi caracterizado pelos educadores como o ponto em que a consciência ambiental já correspondia plenamente a práxis ambiental. E nesse mesmo démarche, o grupo que correspondeu ao que executaram a dinâmica lúdica da competição, o ponto de inflexão foi mais cedo, comprovando a potencialidade que o lúdico traz as metas estabelecidas, comportamento observado na literatura (HUDGENS et al., 2017; SANTANA; PETROVA, 2016).

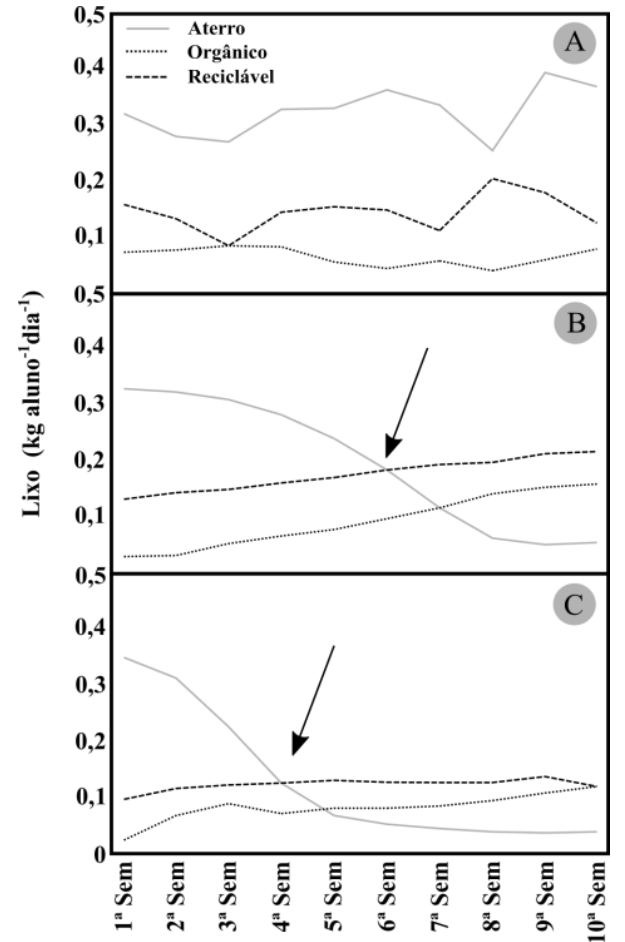

Figura 5. Massa de lixo descartado, dado na linha do tempo, classificado em reciclável, orgânico e que foi destinado aos aterros, na Escola, nos três grupos amostrais: (A) Grupo Controle, (B) Grupo das Metas, e (C) Grupo Lúdico. $\longrightarrow$ Ponto de inflexão, em que a massa de lixo destinada aos aterros foram menor do que para as outras classificações do lixo: reciclável e orgânico.

Ao final das dez semanas, a produção do lixo frente a sua classificação (Figura 6) certificou a mudança de comportamento dos alunos na escola. Houve uma redução no consumo total de resíduos que são destinados a aterros $(p<0,001)$, o valor não foi próximo de zero porcento, mas pela tendência em curto prazo (dez semanas), com a continuidade do projeto esse valor poderá chegar aos resultados próximos pretendido pelos programas "Zero Waste" pelo mundo $(0 \%)$.

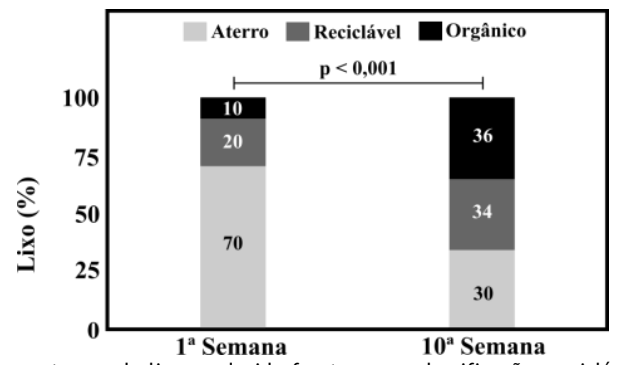

Figura 6. Porcentagem de lixo produzido frente a sua classificação: reciclável, orgânico ou que tiveram destino aos aterros na $1^{\mathrm{a}}$ semana e na $10^{\mathrm{a}}$ semana do projeto.

$\mathrm{Na}$ avaliação desse projeto de atuação na escola pelos educadores, os pesos dados nos critérios da CAPES foram excelentes (> 8; Figura 7), ou seja, o produto educacional (lixeira) como meio ajudou a atingir as finalidades de redução da produção de lixo (implicação socioambiental), de aprimoramento do ensino local (apoio docente), e de aprimoramento da aprendizagem (engajamento dos alunos no processo informativo, formativo e na práxis). A avaliação positiva nesses critérios da CAPES são determinantes para o cumprimento das metas dos projetos de atuação (MELO et al., 2018). 


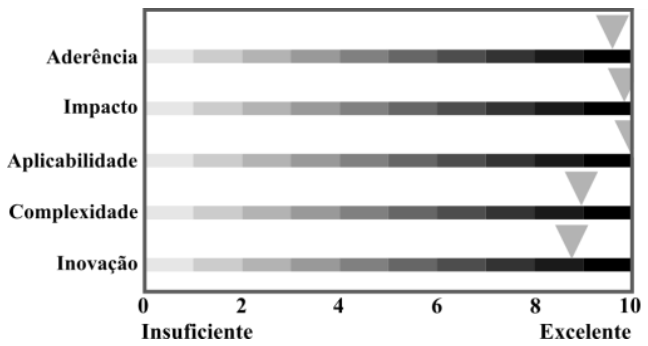

Figura 7. Escala Likert e peso médio dado pelos 21 educadores aos cinco critérios de avaliação do produto técnico e tecnológico da CAPES.

A análise de SWORT, análise com respostas abertas realizadas pelos educadores, foi sintetizada em 17 démarches, sendo que 10 para a força do produto e sua implementação, cinco para oportunidades geradas, um para possível fraqueza, e uma possível ameaça da lixeira (Tabela 1). Esse resultado foi positivo e os aprimoramentos a serem executados não são complexos e não comprometem a importância da lixeira, como registrado em outro trabalho (SANTANA et al., 2018).

Tabela 1. Análise de SWOT da implementação das Lixeiras Informativas.

\begin{tabular}{ll}
\hline \multicolumn{1}{c}{ Análise } & \multicolumn{1}{c}{ Zero Lixo } \\
& 1. Cooperação Coletiva por uma Meta \\
2. Competição Positiva em que todos saem vitoriosos \\
3. Mudança de Percepção dos alunos frente ao Dado \\
4. Aumento do consumo de alimentos que não são embalados e \\
processados
\end{tabular}

\section{CONCLUSÕES}

A hipótese desse projeto de atuação foi aceita, ou seja, que a partir da informação da quantidade de lixo que se foi produzido por cada indivíduo (tomada de consciência), em um determinado ambiente coletivo, houve a redução da sua produção (tomada de práxis). A Lixeira Informativa como produto educacional se mostrou efetiva para as finalidades propostas: ambiental, de ensino e de aprendizagem, havendo potencial para expansão a outros espaços formais de ensino. A dinâmica lúdica foi outra potencialidade, com ela, as finalidade ocorriam em menos tempo. Houve uma mudança comportamental dos alunos de forma perene ao final do projeto, ou seja, houve uma internalização da práxis ambiental a partir da consciência ambiental.

\section{REFERÊNCIAS}

ADAMS, M. A.; BRUENING, M.; OHRI-VACHASPATI, P.; HURLEY, J. C. Location of School Lunch Salad Bars and Fruit and Vegetable Consumption in Middle Schools: A Cross-Sectional Plate Waste Study. Journal of the Academy of Nutrition and Dietetics, v. 116, n. 3, p. 407-416, 2016. Doi: 10.1016/j.jand.2015.10.011 BOEHME, J. S.; LOGOMARSINO, J. V. Reducing Food Waste and Increasing Fruit and Vegetable Consumption in Schools. Health Behavior and Policy Review, v. 4, n. 3, p. 282-293, 2017. Doi: 10.14485/HBPR.4.3.9

CAPES - Coordenação de Aperfeiçoamento de Pessoal de Nível Superior (2019). Ciências Ambientais - Área de Avaliação. Disponível em: https: //goo.gl/FuhCDN Acesso em Abril de 2019.
CIACCI, L.; VASSURA, I.; CAO, Z.; LIU, G.; PASSARINI, F. Recovering the "new twin": Analysis of secondary neodymium sources and recycling potentials in Europe. Resources Conservation and Recycling, v. 142, p. 143-152, 2019. Doi: 10.1016/j.resconrec.2018.11.024

CONNELL, K. Y. H. Internal and external barriers to eco-conscious apparel acquisition. International Journal of Consumer Studies, v. 34, n. 3 , p. $279-286,2010$. Doi: 10.1111/j.14706431.2010.00865.x

COSTELLO, C.; MCGARVEY, R. G.; BIRISCI, E. Achieving Sustainability beyond Zero Waste: A Case Study from a College Football Stadium. Sustainability, v. 9, n. 7, p. 12-36, 2017. Doi: $10.3390 /$ su 9071236 .

GROGAN, P. L. Zero waste: Is Ecotopia possible? Biocycle, v. 38 , n. 1, p. 86-86, 1997.

HOORNWEG, D.; BHADA-TATA, P.; KENNEDY, C. Environment: Waste production must peak this century. Nature, v. 502 n. 7473, p. 615-617, 2013. Doi: $10.1038 / 502615^{\mathrm{a}}$

HUDGENS, M. E.; BARNES, A. S.; LOCKHART, M. K.; ELLSWORTH, S. C.; BECKFORD, M.; SIEGEL, R. M. Small Prizes Improve Food Selection in a School Cafeteria Without Increasing Waste. Clinical Pediatrics, v. 56, n. 2, p. 123-126, 2017. Doi: $10.1177 / 0009922816677546$

IBGE - Instituto Brasileiro de Geografia e Estatística. SIDRA. Disponível em: http://www.ibge.gov.br Acesso em: Abril 2019.

IPEA - Instituto de Pesquisa Econômica Aplicada. Ipea Data. Disponível em: http: / /www.ipea.gov.br Acesso em: Abril 2019.

FORTUNA, L. M.; CASTALDI, M. J. New York City's Reuse Impact Calculator: Quantifying the zero waste impact of materials reuse. Waste Management \& Research, v. 36, n. 12, p. 1190-1200, 2018. Doi: $10.1177 / 0734242 \times 18802623$

KABERA, T.; WILSON, D. C.; NISHIMWE, H. Benchmarking performance of solid waste management and recycling systems in East Africa: Comparing Kigali Rwanda with other major cities. Waste Management \& Research, v. 37, p. 59-72, 2019. Doi: 10.1177/0734242X18819752

KOWALEWSKA, M. T.; KOLLAJTIS-DOLOWY, A. Food, nutrient, and energy waste among school students. British Food Journal, v. 120, n. 8, p. 1807-1831, 2018. Doi: 10.1108/BFJ-11-2017-0611

LIKERT, R. A technique for the measurement of attitudes. Archives of Psychology, v. 22, p. 140-155, 1932.

LIU, Y.; CHENG, S. K.; LIU, X. J.; CAO, X. C.; XUE, L.; LIU, G. Plate Waste in School Lunch Programs in Beijing, China. Sustainability, v. 8 , n. 12, 12-88, 2016. Doi: 10.3390/su8121288

MANOMAIVIBOOL, P.; SRIVICHAI, M.; UNROJ, P. Chiang Rai Zero Waste: Participatory action research to promote source separation in rural areas. Resources Conservation and Recycling, v. 136, n. 9, p. 142-152, 2018. Doi: 10.1016/j.resconrec.2018.04.002

MASON, I. G.; BROOKING, A. K.; OBERENDER, A.; HARFORD, J. M.; HORSLEY, P. G. Implementation of a zero waste program at a university campus. Resources Conservation and Recycling, v. 38, n. 4, p. 257-269, 2003. Doi: 10.1016/S0921-3449(02)00147-7

MELO, R. B. DE; LIMA, C. DE ; DUARTE, C. V. DE M. C.; SOUZA, S. C. DE; SANTANA, O A. Biofísica Ambiental do Semiárido: Quadro Paradidático para Educação Básica. In: Encontro Anual da Biofísica 2018, 2018, Pernambuco. Blucher Biophysics Proceedings. São Paulo: Editora Blucher, 2018. p. 5-8. Doi: 10.5151/biofisica2018-02 NETO, I. F. F.; SOARES, H. M. V. M. Sequential separation of Ag, Al, $\mathrm{Cu}$ and $\mathrm{Pb}$ from a multi-metal leached solution using a zero waste technology. Separation Science and Technology, 53, n. 18, p. 2961-2970, 2018. Doi: 10.1080/01496395.2018.1482342

NEW YORK CITY. Zero Waste Schools. Disponível em https://www1.nyc.gov/assets/zws/index.shtml Acesso em: Abril de 2019. 
PASRICHA, A.; GREENINGER, R. Exploration of 3D printing to create zero-waste sustainable fashion notions and jewelry. Fashion and Textiles, v. 5, n. 30, p. 12-26, 2018. Doi: 10.1186/s40691-0180152-2

PIAGET, J. Part I: Cognitive development in children: Piaget development and learning. Journal of research in science teaching, v. 2, n. 3, p. 176-186, 1964.

SANTANA, O. A.; MELO, R. B.; SOUZA, S. C. DE; DUARTE, C. V. de M. C.; LIMA, C.; COSTA, V. S. O. Paperless e Pegada Hídrica: Zero Papel em uma disciplina do PROFCIAMB. In: Seminário Nacional de Integração da Rede PROFCIAMB, 2018, Belém. Anais do 3. Seminário nacional de integração da rede PROFCIAMB. São Carlos: EESC-USP, 2018. v. 1. p. 1-11.

SANTANA, O. A.; SANTOS, N. K. B.; SILVA, M. M. DA; MORAIS, R. L. DE; ENCINAS, J. I. Árvores potenciais a danos urbanos: manejo através da tecnologia, educação e mobilização social. Revista Tecnologia e Sociedade, v. 11, p. 71-88, 2015. Doi: http://dx.doi.org/10.3895/rts.v11n23.2748

SANTANA, O. A.; PETROVA, Y. Ludicidade no Ensino da Normalidade em um Ambiente Florestal. Inter-ação, v. 41, p. 525544, 2016. Doi: 10.5216/ia.v41i3.41502

SANTANA, O. A. Ensino de ciências em Braille com histórias em quadrinhos roteirizados por cegos. Linhas Críticas (UnB), v. 20, p. 711-743, 2014. Doi: 10.5965/15164896v20n432014711

SCHUCH, A.; MORSCHECK, G.; LEMKE, A.; NELLES, M. Bio-Waste Recycling in Germany - Further Challenges. Compost Science \& Utilization, v. 25, p. S53-S60, 2017. Doi: 10.1080/1065657X.2017.1395716

SCHUPP, C. L.; GETTS, K. M.; OTTEN, J. J. An evaluation of current lunchroom food waste and food rescue programs in a Washington state school district. Journal of Agriculture Food Systems and Community Development, v. 8, n. 1, p. 167-186, 2018. Doi: 10.5304/jafscd.2018.081.013

SHANKS, C. B.; BANNA, J.; SERRANO, E. L. Food Waste in the National School Lunch Program 1978-2015: A Systematic Review. Journal of the Academy of Nutrition and Dietetics, v. 117, n. 11, p. 1792-1807, 2017. Doi: 10.1016/j.jand.2017.06.008

STEINMETZ, V.; VILLAIN-GAMBIER, M.; KLEM, A.; GAMBIER, F.; DUMARCAY, S.; TREBOUE, D. Unveiling TMP Process Water Potential As an Industrial Sourcing of Valuable Lignin-Carbohydrate Complexes toward Zero-Waste Biorefineries. ACS Sustainable Chemistry \& Engineering, v. 7, n. 6, p. 6390-6400, 2019. Doi: 10.1021/acssuschemeng. 9 b00181

SUDARIO, G. Zero Waste Emergency Medicine: A Visual Aid for Clinical Decision Rules in Minor Head Trauma Injury Patients. Annals of Emergency Medicine, v. 72, n. 4, p. S157-S158, 2018. Doi: 10.1016/j.annemergmed.2018.08.410

THE WORD BANK. Urban Development. Disponível em https: / /www.worldbank.org/Acesso em: Abril 2019

UNIVERSITY OF LOUISIANA AT LAFAYETTE. Zero Waste Ragin' Cajun Gameday. Disponível em https://sustainability.louisiana.edu/zerowaste-ragin-cajun-gameday Acesso em: Abril de 2019.

WHYTE, D.; DIDKOVSKY, N.; HUTZLER, S. Zero Waste: Mapping the Evolution of the Iterative Sight-Reading of a Piano Score. Music Theory Spectrum, v. 40, n. 2, p. $302-313,2018$. DOI: $10.1093 / \mathrm{mts} / \mathrm{mty} 019$

VYGOTSKY, L.S. Formação social da mente. São Paulo: Martins Fontes, 1984.

ZANTINGH, M. Wildness and Windsor: Di Brandt's "Zone: < le Detroit $>$," Environmental Praxis, and Urban Nature. ISLEInterdisciplinarity Studies in Literature and Environment, v. 25, n. 2, p. 396-411, 2018. Doi: 10.1093/isle/isy048

ZAR, J. H. Biostatistical analysis. Pearson Education: New York, 1999. 